\title{
Identification of Novel Mutations of the CLCN-1 and SCN4A Genes in Non- dystrophic Myotonia in China
}

Yanxin Meng (D maolv2008@126.com)

Department of prenatal diagnostic center, Shijiazhuang gynaecology and obstertrics Hospital, Hebei, Shijiazhuang 050071, P.R.China

Chunmiao Liu

Shijiazhuang gynaecology and obstertrics Hospital

Mei Yu

Shijiazhuang gynaecology and obstertrics Hospital

Haijuan Zhang

Shijiazhuang gynaecology and obstertrics Hospital

Yuxiu Yang

Shijiazhuang gynaecology and obstertrics Hospital

\section{Research Article}

Keywords: Non-dystrophic myotonias (NDMs), CLCN-1, SCN4A, novel mutations

Posted Date: January 18th, 2021

DOI: https://doi.org/10.21203/rs.3.rs-143997/v1

License: (c) (7) This work is licensed under a Creative Commons Attribution 4.0 International License. Read Full License 


\section{Abstract}

Background:The aim of our study was to characterize the genetic, pathological and clinical alterations of 17 patients in China presenting with non-dystrophic myotonia (NDM).

Methods: We first sequenced the $C L C N-1$ gene in patients having clinical features and muscle pathology indicative of NDM. If no mutations were detected, we subsequently analyzed the SCN4A, KCNE3 and CACNA1S genes. Results: As determined by needle electromyography, patients may have accompanying atypical myopathy as well as muscle hypertrophy, secondary dystonia and joint contracture All participants in this study were administered mexiletine in combination with carbamazepine and showed significant improvements in their myotonia symptoms. Routine pathological examinations showed mild abnormalities in muscle pathology. Oxidative enzyme activity was decreased in many fibers. ATPase studies of fiber subtypes demonstrated a predominance of type 2A fibers and a complete absence of type 2B muscle fibers in patients with $C L C N-1$ mutations. $C L C N-1$ gene mutations were found in 8 cases diagnosed with myotonia congenital by gene screening. The detected mutations included 5 missense, 2 nonsense, 1 deletion and 2 insertions, and these $C L C N-1$ mutations were concentrated in exons 8 and 12. Further gene analysis showed 4 mutations in the SCN $4 A$ gene in patients diagnosed with paramyotonia congenita. One of these mutations was consistent with a previously reported mutation, whereas 3 mutations were novel. All of these novel mutations occurred within "hot spots" of exons 22 and 24. Five patients with NDM lacked any identifiable mutations in CLCN-1, SCN4A, CACNA1S or KCNE3.

Conclusions: Myotonia congenita and paramyotonia congenita are the predominant forms of NDM in China. NDM may be best diagnosed using genetic analysis in combination with clinical features. New mutations of the $C L C N-1$ and SCN4A genes in patients with NDM were detected, we postulate that novel pathogenic genes for NDM occur in China.

\section{Background}

The skeletal muscle chloride ( $C L C N-1)$ and sodium ( $S C N 4 A)$ channels are responsible for Non-dystrophic myotonias (NDMs) which is a group of neuromuscular disorders (prevalence $1: 100000$ ). Delayed muscle relaxation after voluntary or evoked muscle contraction accompanied by atypical muscle pain, weakness and fatigue are common features in this group of myotonias. NDMs are classified as dominant (DMC(OMIM 160800)) and recessive myotonia congenita (RMC(OMIM 255700)), paramyotonia congenita (PMC), hyperkalemic periodic paralysis with myotonia (Hyperpp), hypokalemic periodic paralysis with myotonia (HypoPP), potassium aggravated myotonia (PAM) and a diverse group of sodium channel myotonias ${ }^{[1]}$.

The genetic and phenotypic heterogeneities in NDM create complications in distinguishing patients with sodium channel myotonia from those with myotonia congenita ( $\mathrm{MC}$ ) and underscore the need for these patients to undergo genetic screening. Due to the incomplete dominance of certain mutations with variable penetrance and expression as additional compounding factors, $25 \%$ of the patients with NDM examined in one study lacked an identifiable gene mutation [2] Therefore, we conducted this study to characterize the genetic, skeletal muscle pathology, clinical manifestations of 17 patients with NDM in China.

\section{Methods}

Clinical data

We analyzed 38 people, including 17 patients who had been admitted to the Department of prenatal disgnosis at our hospital from 2015 to 2019 and 5 of their family members. Characteristics of included studies: patients who diagnosed with typical myotonia accompanied by grip or percussion myotonia, an absence of muscular atrophy and an EMG showing typical myotonic discharges; and an absence of myotonic dystrophy.

Skeletal muscle histopathology

After obtaining informed consent from the patients and their family members, muscle biopsy specimens were collected from the biceps brachii. For histopathological analysis, serial frozen sections $(7 \mu \mathrm{m})$ were stained with hematoxylin and eosin (H\&E), modified Gomori trichrome, oil red $\mathrm{O}(\mathrm{ORO})$ and periodic acid-Schiff (PAS) or were treated using histochemical reactions with NADH-TR, succinate dehydrogenase (SDH), adenosine monophosphate (AMP), deaminase, cytochrome C oxidase (COX), acid phosphatase (Acid) and myosin ATPase (pH 4.2, 9.98).

Mutation screening

The genomic DNA was extracted from peripheral blood of 17 patients and their family members. Meanwhile, 100 healthy and unrelated Chinese subjects were selected as the control group. The research of applying Primer Primer 5.0 to design PCR primers which capture the entire coding regions and flanking sequence, included 4 genes: $C L C N-1, S C N 4 A, K C N E 3$ and CACNA1S. 50 ng of genomic DNA with these primers was used a hot-start PCR for amplification of polymorphic markers. PCR products were purified and sequenced using dye terminator chemistry with an ABI Prism 377 DNA Sequencer (Applied Biosystems). The Sequencher 4.90 software was used to analyze the sequences. The relationship between the gene and disease phenotype was analyzed, and a new mutation detected in the 100 control subjects.

\section{Results}

Clinical features analysis

A total of 17 patients were recruited, including 14 boys and 3 girls. Case 1 and case 2 from the same family, and case 3 and 4 from the same family. The age of disease onset was 1-17 years with an average onset of 8 years. Serum CK and $\mathrm{K}^{+}$levels were normal in all 17 patients. All patients suffered from muscular

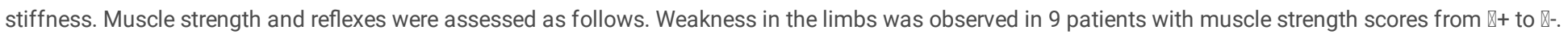




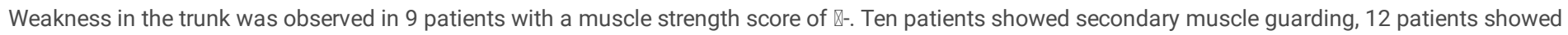
muscular hypertrophy, 3 patients showed scoliosis accompanied by contracture of the Achilles tendon, 1 patient showed contracture of the Achilles tendon and elbow joint and 5 patients showed contracture of only the Achilles tendon. Five patients presented with myotonic discharges accompanied by atypical myopathic discharges upon EMG examination. Three patients had sinus arrhythmia, 1 patient had pre-excitation syndrome, 1 patient had sinus bradycardia and 5 patients had left ventricular enlargement and ventricular myocardial perfusion (Fig. 1, Table 1). There was no patient performance muscle deterioration, testicular atrophy or cataracts. Myotonia was improved by the administration of mexiletine ( $50 \mathrm{mg}, 3$ times/day) in combination with cabamazepine ( $100 \mathrm{mg}$, 2-3 times/day).

Muscle pathology

In the patients with NDMs, the skeletal muscle biopsies showed minor variations in stained with H\&E. The muscle histopathology was characterized by variations in fiber size with the presence of atrophic fibers and by increases in connective tissue elements. Six patients showed occasional degeneration or necrosis, 4 patients exhibited small angular fibers and 2 patients displayed an increase in the number of internal nuclei. Myosin ATPase was characterized by fiber grouping and a predominance of type 2 fibers, which showed that type $2 B$ fibers demonstrated an absence and a predominance of type $2 \mathrm{~A}$ fibers in all patients who with $C L C N-1$ mutations; the type 1 fibers in these patients that either normal or showed mild atrophy. The results from the oxidative enzyme reactions indicated a focal decrease in some fibers with changes predominantly occurring in type 1 fibers (Fig. 2).

Molecular genetic analyses

To obtain an overview of the clinical features, $C L C N-1$ was first sequenced in all patients. Mutations in $C L C N-1$ genes were identified in 8 patients. SCN4A was subsequently sequenced in the remaining 9 patients, and mutations in this gene were identified in 4 patients. The KCNE3 and CACNA1S genes were sequenced in the remaining 5 patients. We were unable to find any mutations in the KCNE3 and CACNA1S genes, although these 5 patients fulfilled the diagnostic criteria for NDMs.

$C L C N-1$ gene mutations in 8 patients

Ten different $C L C N-1$ mutations were identified. A mutation (c.1262_1263insC) detected in one family was consistent with a previously reported mutation. We detected nine novel mutations: five were missense mutations [c.857T>A(p.V286E), c.795A>G(p.D265G), c.1679T>C(p.M560T), c.138C>T(p.R47W) and c.891G>A(p.A298T)]; 2 were nonsense mutations [c.1012C>T(p.R338X) and c.1872G>C(p.E624X)]; 1 was a deletion (c.2330delG); and 1 was an insertion (c.1389_1390ins T). Both the insertion and deletion occurred in exons 8 and 12 . The $C L C N-1$ gene was sequenced in the 5 family members, and we found that the parents of the patients also carried the gene mutation (Figs. 3 - 4).

SCN4A gene mutations in 4 patients

Detection of SCN4A gene was carried and four different mutations were identified, including: 3 were novel mutations [c.5468C>G (p.P1823R), c.5283C>T(p.G1761R) and c.4916 G>A(p.R1639H)]; and 1 was a previously reported mutation [c.3877G>A(p.V1293I)]. These mutations occurred in exons 22 and 24. None of these mutations were found in the chromosomes of the 100 Chinese control subjects. In addition, these mutations were not listed by the 1000 Genomes Project (http://browser.1000genomes.org), which catalogs human genetic variations using 1,197 samples, including 300 East Asian samples (200 Chinese) (Table 2).

\section{Discussion}

NDMs can be easily misdiagnosed due to the fact that it clinical features are common to a host of other myotonia disease which associated with a number of skeletal muscle channelopathies, especially the DMs. As a group of autosomal dominant/recessive disorders caused by mutations in the $C L C N-1$ or $S C N 4 A$ gene, NDMs are caused that delay muscle relaxation after a voluntary or evoked muscle contraction. Clinically, myotonia is the main symptom which affected the muscle system; however, NDMs show marked clinical variability. This suggests that the elucidation of the genetic etiology of these disorders in addition to clinical and electrophysiological features may help to distinguish NDMs from DMs or skeletal muscle diseases with myotonia ${ }^{\text {[3-4] }}$

The average age of disease onset for the 17 patients in our study was 8 years. According to the neurological examinations and EMG evaluations, all of these patients received a clinical diagnosis of NDMs. Muscle biopsies stained with H\&E showed typical myogenic changes with only minor alterations in all samples. The results showing few atrophic fibers, internal nuclei and small angular fibers are consistent with previous studies ${ }^{[5]}$. The differences between NDMs and DMs are that NDMs lack the typical nuclear pyknotic clumps, sarcoplasmic masses, muscle fiber degeneration and severe necrosis of connective tissue. These differences are useful in diagnosing mild cases of myotonic dystrophy, when weakness has not fully developed into pathology. A specific pathological change was found in myosin ATPase. Myosin ATPase characterized by grouping and the predominance of type 2 fibers in the patients. And all patients with $C L C N-1$ gene mutations, type 2B fibers were absent and type 2A fibers were predominant. There are two hypotheses that could explain these phenomena. First, a biochemical abnormality in the nerves of patients with $C L C N-1$ mutations may preclude the development of type $2 \mathrm{~B}$ motor units. The second hypothesis was that due to the repetitive electrical activity associated with myotonia - analogous to that occurring in the conversion of fast muscle fibers to slow fibers by repetitive stimulation of nerves - type 2B fibers become type 2A fibers. This characteristic can be used to distinguishing PMC from MC [6].

Due to "clinical phenotype overlap", the diagnosis of NDMs is dependent on gene analyses. Moreover, numerous studies pointed out that found $C L C N-1$ mutations in $75 \%$ of NDMs suggested that this was a large percentage. By simultaneously sequencing genes about, we detected mutations in most of our patients; $C L C N-1$ gene mutations were found in 8 patients, and $S C N 4 A$ gene mutations in 4 patients. However, we failed to detect any mutations in 5 patients

Page 3/10 
even though they fulfilled the diagnostic criteria for NDMs. It is plausible that deletions or other types of mutations deep within the intron or the promoter region of a gene may underlie the disease in these cases ${ }^{[7.8]}$. Nevertheless, our study showed that the analysis of the genes for $C L C N-1$ together with $S C N 4 A$ resulted in the detection of high levels of mutations in Chinese individuals with NDMs and that it is helpful to also identify the mutations for $K C N E 3$ and CACNA1S in these people with mutations in $C L C N-1$ and $S C N 4 A$. We conclude that the application of second-generation sequencing technology is important in diagnosing NDMs

Eight patients with $C L C N-1$ mutations were clinically diagnosed with MC. Normally, the $C L C N-1$ gene, having a highly conserved domain, encodes the voltagedependent chloride channel CLC1, which is responsible for the large chloride resting potential of skeletal muscle ${ }^{[9]}$. Molecular and genetic researchs on MC showed that skeletal muscle chloride gene mutations ( $C L C N-1)$ mapped to chromosome 7q35. These were "loss of function mutations" that inhibited the depolarization of chloride currents into activated chloride currents to prolong the muscle relaxation process ${ }^{[10]}$. A large number of studies on the mutation expression of $C L C N-1$ in DMC have shown that they could produce an effect on the common gate with dominant negative effect on the wild-type subunit through voltage dependent changes, and the RMC mutation involves a fast gate showing complete loss of two monomer funtions [11]. An explanation of these changes in mutations, which was helpful to understand mutations in the same mutation could lead to both dominant and recessive diseases and why RMC is more seroous ${ }^{[12]}$. In our study, the 2 patients who presented with myotonia showed mild improvement with repetitive activity, and their muscle strength changed slightly without the hypertrophy and tendon reflexes that are characteristic of the DMC gene mutation. Six patients presented with typical myotonia and muscle hypertrophy. Secondary dystonia caused mechanical straining in these patients, and with development of the disease, this could lead to joint contracture and scoliosis, clinical features consistent with RMC. Consequently, the incidence rate in RMC was higher than DMC in our study, and certain clinical manifestations were more common in RMC than in DMC. Some of our patients also presented with accompanying arrhythmia, pre-excitation syndrome or left ventricular enlargement. We postulate that ion channels in cardiomyocytes may contribute to this variable myocardial involvement. To date, more than 100 missense, non-sense, insertions, deletions and splice site mutations have been reported in the $C L C N-1$ gene. The mutations are spread throughout the entire gene and show no location specificity for mutations leading to DMC or RMC [13]. Interestingly, the mutations that we detected in $C L C N-1$ were novel, except for the c.1262 on exon 12, which was reported by Koch [14]. This finding suggested that $C L C N-1$ variations exist for race, region or height. The mutations detected in our study [c.1012C>T(p.R338X), c.1872G>C(p.E6 24X), c.2330delG and c.1389insT] were nonsense, deletion or insertion mutations, which are predicted to cause premature translation termination codons and lead to frame shift or splice site-interrupting mutations. Both of the latter mutations are typically associated with RMC ${ }^{[15]}$. Missense mutations can also lead to either RMC or DMC depending on their location and the effect of the amino acid substitution on channel gating. In our study, five missense mutations were found, with two of these (p.V286E and p.A298T) in $C L C N-1$ occurring in exon 8. Duffield et al. ${ }^{[16]}$ reported that exon 8 encodes the $\mathrm{H}$ and I helix, the $\mathrm{H}-\mathrm{I}$ interlink and part of the $\mathrm{I}-\mathrm{J}$ interlink that form the channel dimer. Thus, mutations occurring in exon 8 affect the formation of the channel dimer, which affects the conductivity of the chloride channel. Both of the missense mutations that we detected in exon 8 have been reported to be compounded with other mutations (nonsense/deletion/insertion/missense). We speculate that these mutations are pathogenic variations because they are truncating mutations. The screening outcome for $C L C N-1$ showed that the form and location of the mutations contribute to the genotype-phenotype relationship at the clinical level.

Four patients who were characterized by marked, typical myotonia exacerbated by cold temperatures, the presence of clear episodes of weakness and with $S C N 4 A$ mutations were clinically diagnosed with PMC. PMC is an autosomal-dominant disorder caused by a mutation in the SCN4A gene, which encodes the a-subunit of the skeletal muscle sodium channel. Normally, this channel is responsible for forming and conducting the action potential. Thus, mutations in SCN4A lead to "gain of channel function" defects, impairing channel inactivation or enhancing channel activation. To date, more than 50 different SCN4A gene mutations have been reported from several populations, and many of these mutations are distributed in exons 13, 19, 22, 23 and 24. Exons 22 and 24 have been recognized as mutation hot-spot regions in PMC [17]. Three novel missense mutations occurred in exon 24, and 1 missense mutation has been reported in exon 22, both of which are located in hot-spot regions. Studies using different gene mutations in the functional domains of the sodium channel complex have confirmed that they form the structural basis for the deactivation mechanism ${ }^{[18]}$. For example, the mutation c.3877G>A(p.V1293I) located in the cytoplasmic region of membrane domains $\varangle / \otimes$ lead to the inactivation of the sodium channel and result in a unique temperature-sensitive phenotype ${ }^{[19]}$. In our study, a twoyear-old girl who carried this mutation showed myotonia that was exacerbated by cold and accompanied by muscle hypertrophy and joint contracture, consistent with a previous report ${ }^{[20]}$. The 3 novel mutations in exon 24 detected in our study were located in the voltage-sensing transmembrane S4 segment in domain $₫$ of the sodium channel and affected the rapid depolarization process. Interestingly, analysis of the family members determined that the myotonia syndrome was relieved with age. This finding suggested that PMC has a mild impact on the quality of life. These findings indicated that single mutations in the SCN4A gene often affect the processes of slowing fast inactivation, impairing slow inactivation, hastening recovery from inactivation and slowing deactivation, eventually leading to different clinical phenotypes. Our findings also suggest that SCN $4 A$ mutations may have height, race and region specificity [21-22].

We were unable to detect any mutations in 5 patients using simultaneous sequencing of $C L C N-1, S C N 4 A, K C N E 3$ and $C A C N A 1 S$. We postulate that mutations or deletions in introns or novel genes may correlate with the disease. Therefore, genetic testing may become the gold standard for the definitive diagnosis of patients with NDM, and in the future, DNA chip technology may replace the time-consuming electrodiagnostic studies currently required in the initial evaluation.

After clinical, electrophysiological, skeletal muscle pathology and genetic analyses, all patients were administered carbamazepine (100 mg, 2-3 times/day) and mexiletine (50 mg, 3 times/day). Clinical symptoms improved significantly, consistent with previously published reports [21,22]. In vitro studies have

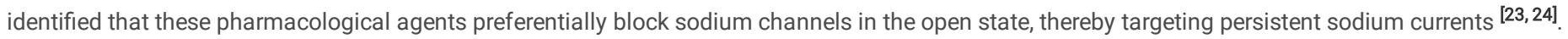

\section{Conclusion}


$\mathrm{MC}$ and PMC have similar but heterogeneous clinical phenotypes. Analysis of the $C L C N-1$ and $S C N 4 A$ genes can identify a large number of mutations in patients with NDMs. Most of the abundant gene mutations detected in $C L C N-1$ and $S C N 4 A$ in our study were novel. In addition, we found a significant racial difference for the mutations in $C L C N-1$ and SCN4A genes. Because new gene mutations are likely to be discovered in patients with NDMs, DNA chip technology will become invaluable for diagnosing NDMs. The administration of carbamazepine combined with mexiletine offered significant benefits to patients, indicating the clinical merit of these drugs.

\section{Abbreviations}

Non-dystrophic myotonias: NDMs, Myotonia congenital: MC, Paramyotnia congenital : PMC, Potassium aggravated myotonia: PAM,Sodium channel myotonias: SCM, Thomsen's myotonia: DMC, Becker's recessive generalized myotonia: RMC

\section{Declarations}

\section{Ethics approval and consent to participate:}

The full name of the ethics committee囚The Medical Ethics Committee of Shijiazhuang No 4 Hospital, the reference number: 20200145

Consent for publication: I and all authors \the undersigned, give my consent for my photograph and/or case history to be published in a BMC Medical Genomic. All procedures performed in studies involving human participants were in accordance with the ethical standards of the institutional and/or national research committee and with the 1964 Helsinki Declaration and its later amendments or comparable ethical standards.

Availability of data and materials: All data generated or analysed during this study are included in this published article [and its supplementary information files].

All experimental protocols were approved by a named institutional and/or licensing committee.

All methods were carried out in accordance with relevant guidelines and regulations.

Competing interests: We declare that we have no conflict of interest.

Founding: This study was supported by grants from the Natural fund for youth of Hebei province (No.H2017106030), the corresponding and the first author is the project leader.

\section{Authors' contributions:}

Yanxin Meng carried out the design and coordinated the study, participated in most of the experiments, prepared the manuscript and agreed for all aspects of the work.

Chunmiao Liu contributed in the conception of the work, conducting the study, revising the draft, approval of the final version of the manuscript, and agreed for all aspects of the work

Mei Yu contributed in the conception of the work, conducting the study for all aspects of the work

Haijuan Zhang approval of the final version of the manuscript, and agreed for all aspects of the work

Yuxiu Yang contributed in the conception of the work

All authors have read and approved the content of the manuscript.

Acknowledgements: The authors thank the participants described in this study for their cooperation and the Shijiazhuang gynaecology and obstertrics Hospital for excellent technical assistance. Thank you for American journal experts Premium editing service. Our research carried out on humans has followed international and national regulations, we have written informed consent to all patients.

\section{Authors' information (optional)]}

\section{*Corresponding author:}

Yanxin Meng* $\otimes P h D$

Department of prenatal diagnostic center, Shijiazhuang gynaecology and obstertrics Hospital Hebei, Shijiazhuang 050071, P.R.China

Tel (0311)85281381

Fax: (0311) 85281381

Email: maolv2008@126.com

1 First author: Yanxin-Meng1; Email:maolv2008@126c.om; Department of prenatal diagnostic center, Shijiazhuang gynaecology and obstertrics Hospital 
2 First author: Chunmiao Liu1; Email: liuchunmiao212327@126.com; Department of obstetrics and gynecology, Shijiazhuang gynaecology and obstertrics Hospital

3 Third author: Mei Yu; Email:yumeicqzd@163.com; Department of prenatal diagnostic center, Shijiazhuang gynaecology and obstertrics Hospital

4 Fourth author: Haijuan Zhang; Email: Zhanghaijuan2020@126.com, Department of obstetrics and gynecology, Shijiazhuang gynaecology and obstertrics Hospital

5 Fifth author: Yuxiu Yang; Email: yangyuxiu@sina.com, Department of obstetrics and gynecology, Shijiazhuang gynaecology and obstertrics Hospital

\section{References}

1. Jaya R. Trivedi, Brian Bundy, Jeffrey Statland, et al. Non-dystrophic myotonia: prospective study of objective and patient reported outcomes. Brain.2013, $1-12$

2. Jeroen Trip, Gea Drost, Dennis J Verbove, et al. In tandem analysis of CLCN1 and SCN4A greatly enhances mutation detection in families with nondystrophic myotonia. European Journal of Human Genetic s.2008,16:921-929

3. Tetsuo Ashizama, Parthas.Sarkar. Myotonic dystrophy types 1 and 2. Handbook of Clinical Neurology. 2011, 101: 194-237

4. Matthews E,Fialho D,Tan SV,et al. The non-dystrophic myotonicas: molecular pathogenesis, diagnosis and treatment. Brain,2010;133: 9-12

5. Carole Hirn , George Shapovalov , Olivier Petermann, et al. Na v1.4 Deregu lation in Dystrophic Skeletal Muscle Leads to Na + Overload and Enhanced Cell Death. The journal of General physiol ogy.2008;132(2):199-208

6. JERRY CREWS, KENNETH K, KAISER, et al. MUSCLE PATHOLOGY OF MY OTONIA CONGENITA. Journal of the Neurological Sciences.1976; 28 : 449 -457

7. Kun wu, xiaoying Wang, Hebin Yao. Mutation status of gene CACNA1S and SCN4A in the hypokalemic periodic paralysis pedigree in Chinese population. Med J china PLA. 2013; 38:302-307

8. Magnus R.Dias Dasilva, Janete M.Cerutti, Liliane a.T.arnaldi, et al. A mutation in th e KCNE3 postassium channel gene is associated with susceptibility to thyrotoxic hy pokalemic periodic paralysis. The Journal of Clinical Endocrinology \& Metabolis m .2002; 87(11):4881- 4884

9. Robert S. Kass. The channelopathies: novel insights into molecular and genetic me chanisms of human disease. The Journal of Clinical.Investigation.2005;115: 1986-1989

10. James A Burge, Michael G Hanna. Novel Insights into the mechanisms of skeleta I muscle channelopathies . Current neurology and neuroscience reports, 2012

11. Robert L. Ruff MD, PhD, Barbara E. Shapiro MD, PhD. Disorders of Skeletal Mus cle Membrane Excitability: Myotonia Congenita, Paramyotonia Congenita, Periodic Paralysis, and Related Syndromes. Neuromuscular Disorders in Clinical Practi ce .2014; pp 1149-1185

12. Trip J, Drost G, Ginjaar HB, Nieman FH, van der Kooi AJ, de Visser M, et al. Redefining the clinical phenotypes of non-dystrophic myotonic syndromes. J Neurol Neurosurg Psychiatry. 2009; 80: 647-52

13. Chen Sun. Clinical and genetic investigations of patients with myotonia congenita in Northern Norway. A dissertation for the degree of Philosophiae Doctor . 2011

14. Christof Meyer-Kleine, Klaus Steinmeyer, Kenneth Ricker, et al. Spectrum of Mutations in the Major Human Skeletal Muscle Chloride Channel Gene (CLCN I) Leading to Myotonia. Am. J. Hum. Genet. 1995;( 57):1325-1334

15. Michael Pusch. Myotonia Caused by Mutations in the Muscle Chloride Channel Gene CLCN1. HUMAN MUTATION. 2002;19:423-434

16. Fialho D, Schorge S, Pucovska U, et.al. Chloride channel myotonia: exon 8 hot-spot for dominant-negative interactions. Brain 2007; 130(Pt 12):3265-74

17. Vicart S $₫$ Sternberg D\&Fontaine B . Human skeletal muscle sodium channelopathies. Neurol Sci,2005,26:194-202

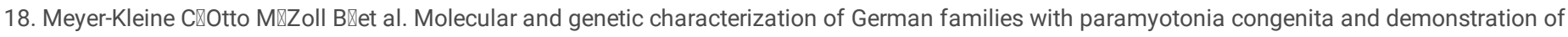
founder effect in the Ravensberg families. Hum Genet,1994,93: 707-10

19. Manuela Koch, Karin Baumbach' Alfred George, et al. Paramyotonia congenital wi thout paralysis on exposure to cold $\mathbb{A}$ A novel mutation in the SCN4A gene (Val I2 93Ile) .NeuroReport,1995, 6ه2001-2004

20. Dipa L,Raja Ray an,Michael G,et al. Skeletal muscle channelopathies: nondystroph ic myotonias and periodic paralysis ? Current Opinion in Neurology, 2010;23: $466-476$

21. Jean-François Desaphy, Anna Modoni, Mauro LoMonaco, et al. Dramatic improve ement of myotonia permanens with flecainide: a two-case report of a possible ben ch-to-bedside pharmacogenetics strategy. Eur J Clinical Pharmac ol .2013;69:1037-1039

22. L. Logigian, W.B. Martens, R.T. Moxley IV, et al. Mexiletine is an effective an timyotonia treatment in myotonic dystrophy type 1. Neurology 2010;74:1 4411448

23. G Meola, M G Hanna, B Fontaine. Channelopathies Diagnosis and new treatme nt in muscle. J.Neurol.Neurosurg.Psychiatry.2009;80:360-365

24. Trip J, Drost GG, van Engelen BGM, et al. Drug treatment for myotonia. The Cochrane Collaboration. 2011;1:1-30

\section{Tables}

Table 1. Clinical features of 17 patients with non-dystrophic myotonia 


\begin{tabular}{|c|c|c|c|c|c|c|c|c|c|c|c|c|}
\hline \multirow[t]{2}{*}{ case } & \multirow[t]{2}{*}{ Gender/Age } & \multicolumn{2}{|l|}{ Myotonia } & \multirow{2}{*}{$\begin{array}{l}\text { Warm-up } \\
\text { phenomena }\end{array}$} & \multirow{2}{*}{$\begin{array}{l}\text { Muscle } \\
\text { volume } \\
\text { of full }\end{array}$} & \multicolumn{2}{|l|}{ EMG } & \multirow{2}{*}{$\begin{array}{l}\text { Hyper- } \\
\text { myotonia }\end{array}$} & \multicolumn{2}{|c|}{ Muscle force } & \multirow{2}{*}{$\begin{array}{l}\text { Cardiac } \\
\text { involvement }\end{array}$} & \multirow{2}{*}{$\begin{array}{l}\text { Col } \\
\text { of }\end{array}$} \\
\hline & & $\begin{array}{l}\text { Percussion } \\
\text { myotonia }\end{array}$ & $\begin{array}{l}\text { Grip } \\
\text { myotonia }\end{array}$ & & & $\begin{array}{l}\text { Myotonic } \\
\text { potential/ }\end{array}$ & $\begin{array}{l}\text { Myopathic } \\
\text { potential }\end{array}$ & & Limbs & trunk & & \\
\hline 1 & $\mathrm{M} / 5$ & + & + & + & + & +++ & - & + & $\nabla-$ & 口 & M & - \\
\hline 2 & $\mathrm{M} / 11$ & + & + & + & - & +++ & - & - & $\square$ & प & $A$ & - \\
\hline 3 & $\mathrm{M} / 1$ & + & + & + & + & +++ & - & - & $\square$ & $\square$ & - & $\operatorname{scc}$ \\
\hline 4 & $M / 5$ & + & + & + & + & ++++ & - & + & $\bigotimes+$ & $\nabla-$ & A & Acl \\
\hline 5 & $\mathrm{M} / 9$ & + & + & + & + & ++++ & Combination & + & $\nabla-$ & $\square$ & - & Acl \\
\hline 6 & $\mathrm{M} / 9$ & + & + & + & + & ++++ & Combination & + & $\nabla-$ & $\square$ & M & - \\
\hline 7 & $\mathrm{~F} / 7$ & + & + & + & + & +++ & Combination & - & $\nabla-$ & $\nabla-$ & - & - \\
\hline 8 & $\mathrm{~F} / 11$ & + & + & + & + & ++++ & - & - & $\otimes-$ & $\square$ & - & $\operatorname{scc}$ \\
\hline 9 & $\mathrm{M} / 8$ & + & + & + & - & ++++ & - & - & प & $\nabla-$ & $A$ & - \\
\hline 10 & $\mathrm{M} / 16$ & + & + & + & + & ++++ & - & - & $\square$ & $\square$ & M & Acl \\
\hline 11 & $M / 15$ & + & + & + & + & +++ & - & - & $\square$ & $\nabla-$ & A & - \\
\hline 12 & $\mathrm{~F} / 2$ & + & + & + & + & ++++ & - & + & $\square$ & $\nabla-$ & M & - \\
\hline 13 & $\mathrm{M} / 3$ & + & + & + & + & +++ & Combination & + & $\bigotimes+$ & $\nabla-$ & - & - \\
\hline 14 & $\mathrm{M} / 3$ & + & + & - & - & ++ & Combination & + & $\square$ & $\nabla-$ & - & Acl \\
\hline 15 & $\mathrm{M} / 10$ & + & + & - & + & +++ & - & - & $\square$ & $\nabla-$ & - & Act \\
\hline 16 & $\mathrm{M} / 15$ & - & + & - & + & +++ & - & + & $\square$ & 口 & - & - \\
\hline 17 & $\mathrm{M} / 17$ & + & & + & + & + & Combination & - & प & प & $A$ & \\
\hline
\end{tabular}

M,male; F, female;-, negative; +, Positive;+ to +++,the degree based on EMG examination; A, arrhythmia; M,myocardiopathy; A,arrhythmia; M,myocardiopathy;

Table 2. CLCN-1 and SCN4A gene mapping of patients with non-dystrophic myotonia

\begin{tabular}{|c|c|c|c|}
\hline Patient No. & Exon & Gene & Mutations \\
\hline 1 & $8 ; 9$ & CLCN-1 & c. $857 \mathrm{~T}>\mathrm{A} ; \mathrm{c} .1012 \mathrm{C}>\mathrm{T}$ \\
\hline 2 & $8 ; 9$ & $C L C N-1$ & c. $857 T>A ; c .1012 C>T$ \\
\hline 3 & 15 & CLCN-1 & c. $1679 \mathrm{~T}>\mathrm{C}$ \\
\hline 4 & 12 & $C L C N-1$ & c. 1262 insC \\
\hline 5 & 12 & CLCN-1 & c. 1262 insC \\
\hline 6 & $12 ; 19$ & $C L C N-1$ & c.1389insT; c. $2330 \mathrm{delG}$ \\
\hline 7 & $7 ; 16$ & $C L C N-1$ & c. $795 \mathrm{~A}>\mathrm{G} ; \mathrm{c} .1872 \mathrm{G}>\mathrm{T}$ \\
\hline 8 & $1 ; 8$ & $C L C N-1$ & c. $138 \mathrm{C}>\mathrm{T} ; \mathrm{c} .891 \mathrm{G}>\mathrm{A}$ \\
\hline 9 & 24 & $S C N 4 A$ & c. $4916 \mathrm{G}>\mathrm{A}$ \\
\hline 10 & 24 & $S C N 4 A$ & c. $5283 \mathrm{C}>\mathrm{T}$ \\
\hline 11 & 24 & $S C N 4 A$ & c. $5468 \mathrm{C}>\mathrm{G}$ \\
\hline 12 & 22 & $S C N 4 A$ & c. $3877 \mathrm{G}>\mathrm{A}$ \\
\hline
\end{tabular}

\section{Figures}




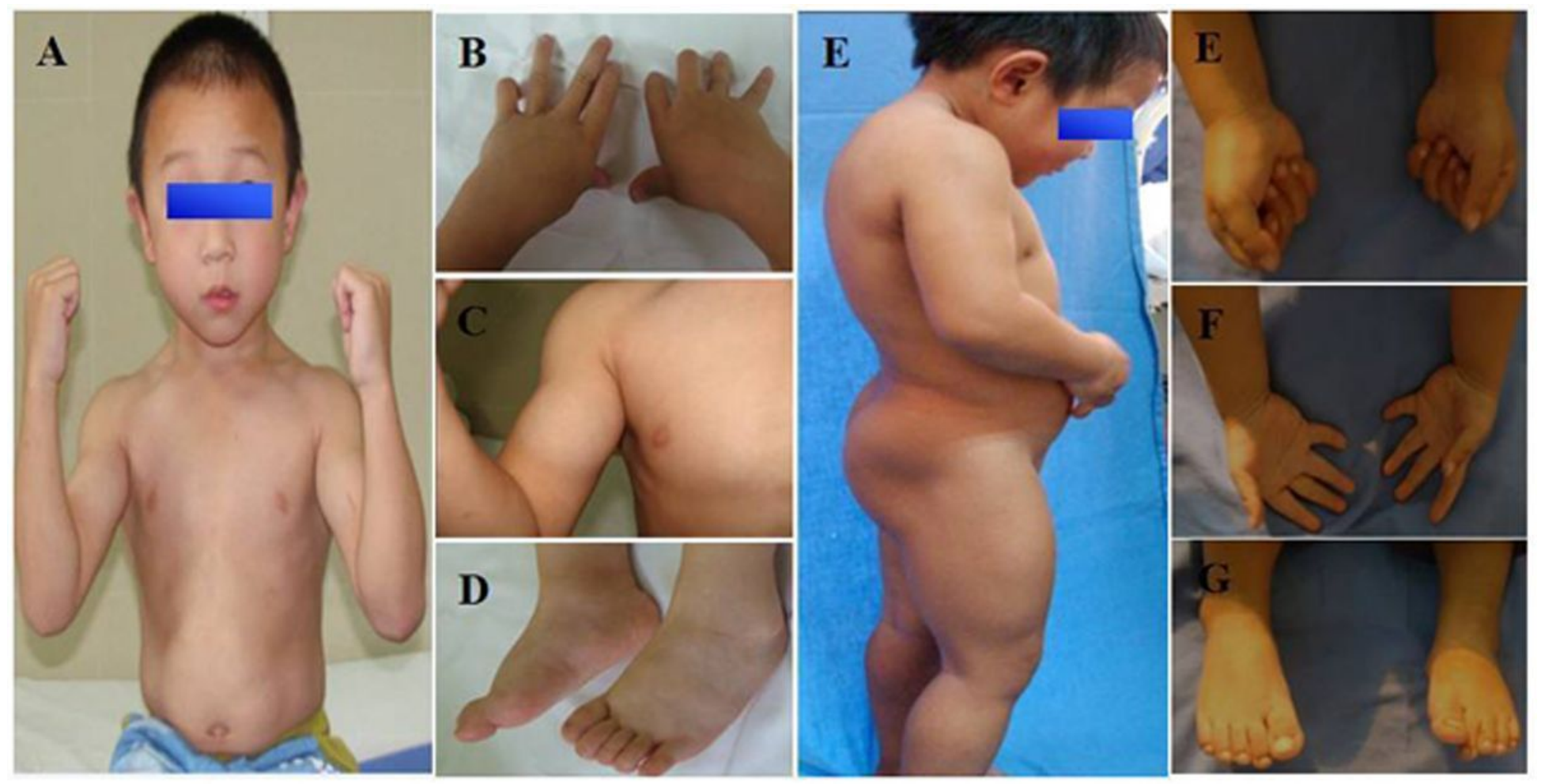

\section{Figure 1}

Clinical features of non-dystrophic myotonia. Left (A-D): A patient with MC showing muscle stiffness, muscular hypertrophy and grip myotonia. Right (E-G): A patient with PMC showing muscle stiffness, joint contracture and muscular hypertrophy with lower limbs affected.

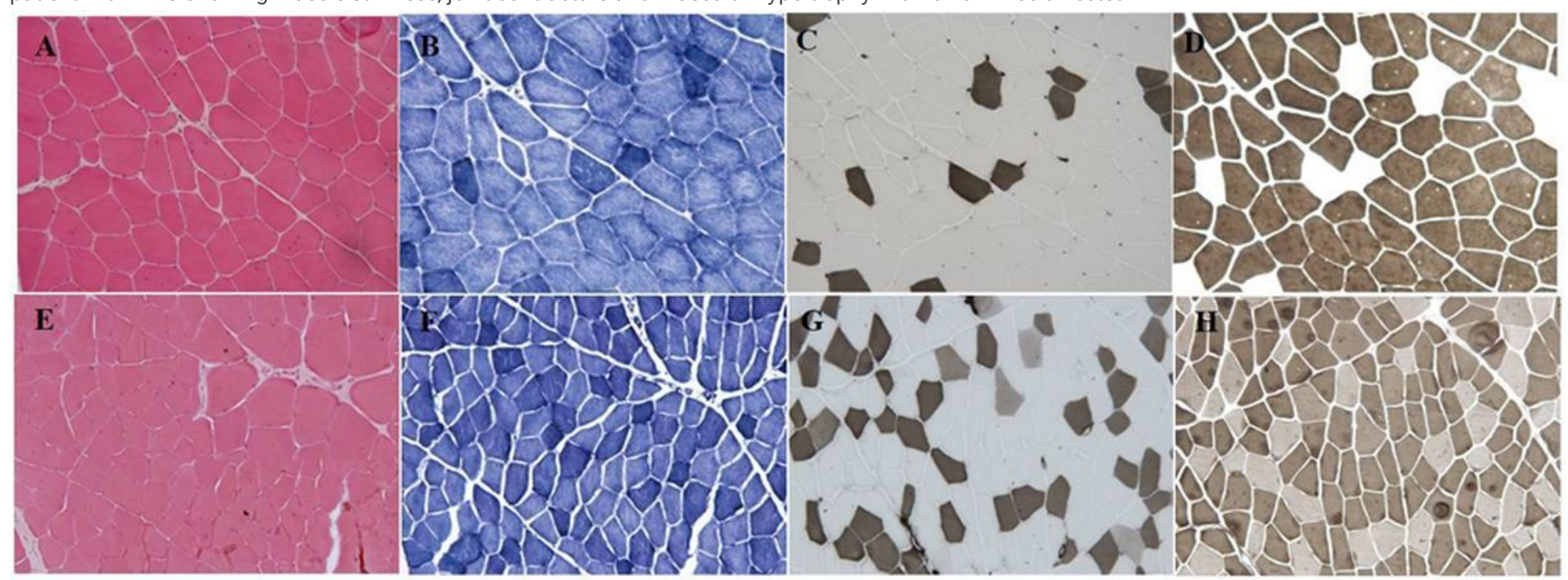

\section{Figure 2}

Histologic characteristics of non-dystrophic myotonias. A-D $(\times 100)$ are sections of muscle biopsied from patients with $\mathrm{CLCN}-1$ mutations; $\mathrm{E}-\mathrm{H}(\times 100)$ are from patients with SCN4A mutations. H\&E staining shows the variability in the diameter of the muscle fibers. Internal nuclei and small angular fibers are visible, and connective tissue elements are mildly increased $(A, E)$. The NADH-TR staining highlights focal decreases and a ragged appearance of the fibers (B, F). $\mathrm{C}-\mathrm{H}$ are from modified ATPase reactions $(\mathrm{pH}=4.2,9.98)$. Following preincubation, small angular fibers and two types of fibers can be observed, although type 2 fibers are predominant; in addition, type 2B fibers were deficient in patients with CLCN-1 mutations $(G)$. 


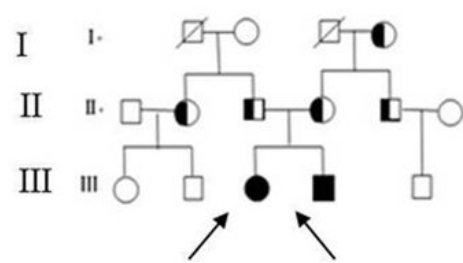

Pedigree 1

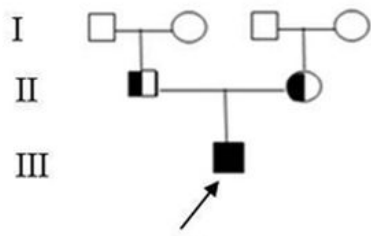

Pedigree 2
Patient1,2 Nham

Carrier

Wild type

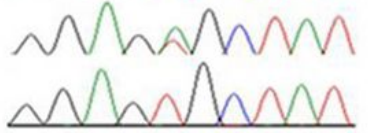

Exon8 c.857 T >A

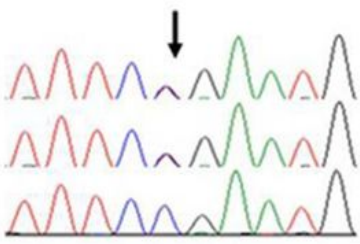

Exon9 c.1012C $>$ T .

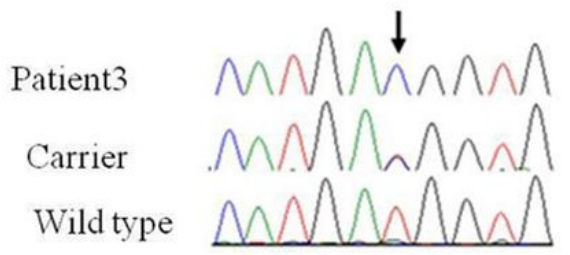

Exon15 c. $1679 \mathrm{~T}>\mathrm{C}$.

Patient 4.5

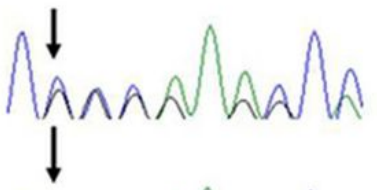

Wild type

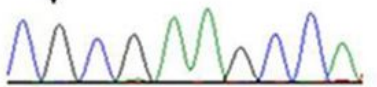

Exon12 c.1262insC .

-Pedigree 3

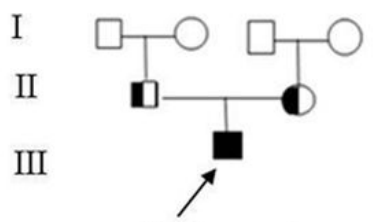

Pedigree 4

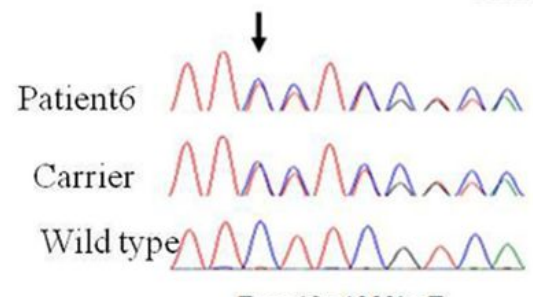

Exon12c.1389insT,

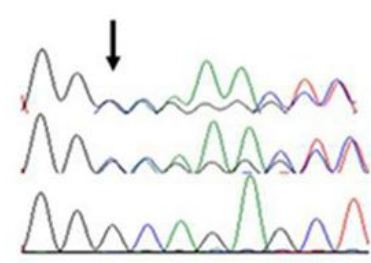

Exon19 c.c.2330delG

\section{Figure 3}

Pedigree 12 from 3 generations of one family with CLCN-1 mutations. Right: Confirmation of heterozygous mutations in the CLCN-1 gene; T>A mutation at nucleotide 857 in the mother, and C>T mutation at nucleotide 1012 in the father; Pedigree 2 from 3 generations of one family with CLCN-1 gene mutations. Right: Confirmation of a homozygous mutation in CLCN-1, T>C mutation at nucleotide 1679 in the mother and father; Pedigree 3 from 4 generations of one family with CLCN-1 gene mutations. Right: Confirmation of homozygote mutations in CLCN-1; C inserted at nucleotide 1262 in the mother, uncle and grandmother; Pedigree 4 from 3 generations of one family with CLCN-1 gene mutations. Right: Confirmation of a novel homozygous mutation in CLCN-1, T inserted mutation at nucleotide 1389 in the mother and mutation c.2330delG in the father 


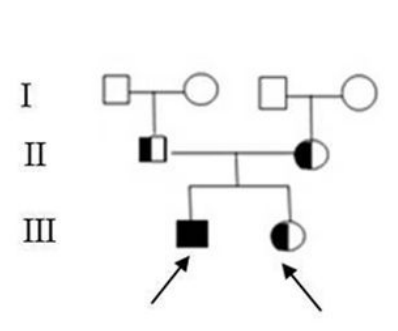

Patient7 Narrier Mm

MA

MAnMMN

wild type MWWMM

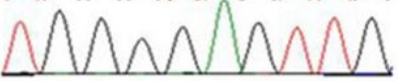

Exon7c.795A>G

Exon16 .1872G $>\mathrm{T}$,

Patient8
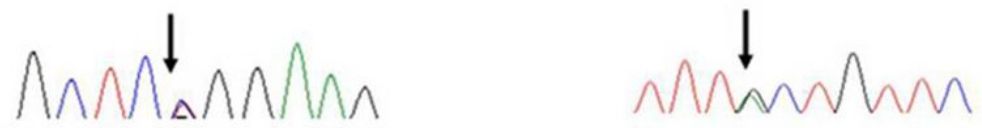

Wild type $\Delta \Delta M \Delta \sim \triangle M$

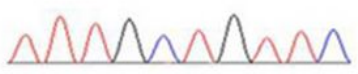

Exon1 c. $138 \mathrm{C}>\mathrm{T}$.

Exon8 $891 \mathrm{G}>\mathrm{A}$

Patient9 MMnN M

Patient10

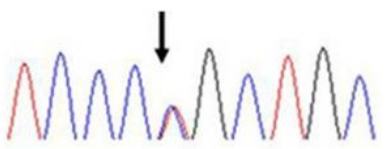

Wild type $\frac{\Delta \text { Exon24 c. 4916G>A. }}{\Delta(M ⿻}$

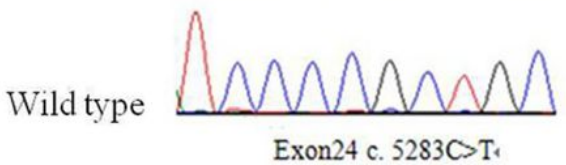

Patient11 $\cap \backsim \backsim \backsim$

Patient12 $\sqrt{ }$

Wild type $\frac{\Lambda \cup \cup v}{\text { Exon24 c.5468 }>\mathrm{G}}$

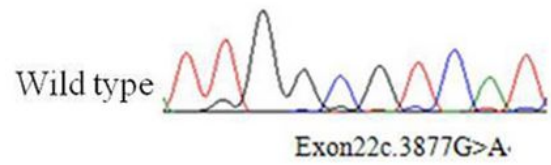

\section{Figure 4}

Pedigree 5 from 3 generations of one family with CLCN-1 gene mutations. Right: Confirmation of novel heterozygous mutations in CLCN-1; $>>\mathrm{G}$ mutation at nucleotide 795 in the mother, and G>C mutation at nucleotide 1872 in the father. Sporadic case: Confirmation of heterozygous mutations in the CLCN-1gene: C>T mutation at nucleotide 138 in one patient, and G>A mutation at nucleotide 891 in one patient. Pedigrees and sequence analyses from families with PMC: Left: Confirmation of a novel heterozygous mutation in the SCN4A gene; G>A mutation at nucleotide 4916 in one patient. Right: Confirmation of a novel heterozygous mutation in the SCN4A gene; C>T mutation at nucleotide 5283 in one patient. Left: Confirmation of a novel heterozygous mutation in the SCN4A gene; $C>G$ mutation at nucleotide 5468 in one patient. Right: Confirmation of a novel heterozygous mutation in SCN4A; G>A mutation at nucleotide 3877 in one patient. 